OPEN ACCESS

Edited by:

Rouzbeh Motiei-Langroudi, University of Kentucky, United States

Reviewed by: Bharat Guthikonda, Louisiana State University Health Shreveport, United States Rushna Ali, Spectrum Health, United States

*Correspondence: Cunzu Wang neurosurgeonwang@126.com

$$
\begin{array}{r}
\text { Specialty section: } \\
\text { This article was submitted to } \\
\text { Neurotrauma, } \\
\text { a section of the journal } \\
\text { Frontiers in Neurology }
\end{array}
$$

Received: 09 November 2021 Accepted: 13 December 2021 Published: 28 January 2022

Citation:

Wei $Z$, Jiang $H$, Wang $Y$ and Wang $C$ (2022) Effect of Twist-Drill Craniostomy With Hollow Screws for Evacuation of Chronic Subdural Hematoma: A Meta-Analysis.

Front. Neurol. 12:811873. doi: 10.3389/fneur.2021.811873

\section{Effect of Twist-Drill Craniostomy With Hollow Screws for Evacuation of Chronic Subdural Hematoma: A Meta-Analysis}

\author{
Zeng Wei ${ }^{1}$, Haixiao Jiang ${ }^{1}$, Ying Wang ${ }^{1}$ and Cunzu Wang ${ }^{2 *}$ \\ ${ }^{1}$ Medical College of Yangzhou University, Yangzhou University, Yangzhou, China, ${ }^{2}$ Northern Jiangsu People's Hospital \\ (NJPH), Yangzhou, China
}

Objective: This study systematically reviews the clinical efficacy and safety of twist-drill craniostomy with hollow screws in chronic subdural hematoma treatment.

Methods: A computerized search of PubMed, Embase, Web of Science, Cochrane Library, World Health Organization International Trial Registry platform, CBM, CNKI, and Wanfang Database was performed to retrieve randomized controlled trials or case-control trials using twist-drill craniostomy (TDC) with hollow screws for the evacuation of chronic subdural hematoma from the date of databases' inception to July 2021. Two investigators independently screened the studies and extracted data in strict accordance with pre-established inclusion and exclusion criteria. RevMan 5.3 software or STATA was used for meta-analysis after evaluating the methodological quality of the included studies.

Results: A total of 4 randomized controlled trials and 16 case-control trials with a total of 2,536 cases were included. Results of the meta-analysis showed that the surgical success rate and postoperative recurrence rate of TDC with hollow screws were slightly higher compared to the burr hole craniostomy $(\mathrm{BHC})$ group, but showed no statistical significance $(\mathrm{RR}=1.03, P=0.05 ; \mathrm{RR}=1.13, P=0.50)$. However, subgroup analysis showed that the use of $\mathrm{YL}-1$ needle had a higher success rate and lower recurrence rate $(\mathrm{RR}=1.05, P=0.02<0.05 ; \mathrm{RR}=0.584, P=0.002)$, and TDC with hollow screws had a lower incidence rate of postoperative complications and postoperative acute intracranial hemorrhage compared with $\mathrm{BHC}$, also revealing an overall shorter hospital stay $(\mathrm{RR}=0.57, P=0.0002<0.05 ; \mathrm{RR}=0.584, P=0.027<0.05 ; \mathrm{WMD}=-3.752$, $P<0.001)$. However, the postoperative mortality rate was practically the same between the two groups $(\mathrm{OR}=1.01, P=0.95>0.05)$.

Conclusion: Twist-drill craniostomy with hollow screws is not inferior or superior to $\mathrm{BHC}$ in efficacy, and this strategy is safer and minimally invasive, which is reflected in a lower incidence of acute intracranial hemorrhage, overall complication rate, and length of hospital stay.

Systematic Review Registration: https://www.crd.york.ac.uk/prospero/, identifier: CRD42021270835.

Keywords: SEPs, the hollow screw, YL-1 needle, chronic subdural hematoma, meta-analysis 


\section{INTRODUCTION}

Chronic subdural hematoma $(\mathrm{CSDH})$ is one of the most common diseases encountered in the neurosurgical department. The incidence of CSDH has been reported to be $1.72-20.60$ per 100,000 people, and the elderly population accounts for the large majority. Moreover, due to the aging of the global population, some scholars have observed an increased incidence of CSDH (15). The main feature of CSDH is the accumulation of blood and its by-products in the subdural space; therefore, the progression of $\mathrm{CSDH}$ is relatively slow (6). The surgical evacuation of hematoma remains the primary treatment method of CSDH. There are three commonly used surgical methods in clinical practice to date, including burr hole craniostomy (BHC), twistdrill craniostomy (TDC), and craniotomy. BHC and TDC are used as primary options, while craniotomy is generally used for acute subdural hematoma or extensive CSDH and is considered as a secondary option for most patients with $\operatorname{CSDH}(7,8)$. There is still a debate on whether BHC or TDC is better. The evidencebased studies by Weigel et al. (9) and Lega et al. (10) suggested that $\mathrm{BHC}$ is safer and more effective than TDC. However, with the improvement of TDC, especially TDC with hollow screws connected to a closed drainage system has emerged. TDC with hollow screws, a bedside procedure, utilizes a special puncture needle to drill and penetrate the skull and dura mater directly to the hematoma cavity, and the closed drainage system is reconnected through the twist-drill hole on the skull without the need to insert a catheter into the subdural space. There is increasing evidence that TDC with hollow screws should be the first-line intervention clinically, but most of the published data are from single institutional experiences. There are three kinds of puncture needles currently available: the Subdural Evacuating Port System (SEPS, initially manufactured by Medical Designs LLCA and later by Medtronic Inc.), the hollow screw (initially manufactured by Fehling Instruments and later by Teleflex Medical), and YL-1 needle (manufactured by Beijing WanTeFu Medical Apparatus). Although Hoffman et al. (11) and Chari et al. (12) have done evidence-based research on the TDC with hollow screws, their studies were on SEPS only. Moreover, the study by Chari et al. is relatively old and did not include the findings on TDC that utilized YL-1 needles. Furthermore, both did not include the results of randomized controlled trials. Hence, this study systematically reviews the clinical efficacy and safety of TDC with hollow screws (including SEPS, the hollow screw, and YL-1 needle) in CSDH treatment, thereby providing a reference for clinical diagnosis and treatment.

\section{MATERIALS AND METHODS}

\section{Search Strategy}

A systematic literature search of PubMed, Embase, Web of Science, Cochrane Library, World Health Organization International Trial Registry platform, CBM, CNKI, and Wanfang Database was performed. Keywords used included "Hematoma, Subdural, Chronic, Craniotomy, YL-1 needle, hollow screws, minimally invasive puncture drainage, Twist-drill craniostomy, subdural evacuating port system, SEPS, randomized controlled trial, and Case-Control Studies." The search was performed by combining subject headings with free words, and the publication time was not restricted. The search strategy was determined after multiple independent pre-searches by two investigators.

\section{Study Selection}

Inclusion criteria included: (1) study design: the trial was designed as a randomized controlled trial or case-control trial with more than 10 patients (prospective and retrospective). The intervention was a primary procedure to treat $\mathrm{CSDH}$ with TDC with hollow screws (i.e., SEPS, the hollow screw, and the YL-1 needle); (2) subjects: any patient with chronic or subacute subdural hematoma (imaging diagnosis: mainly crescentic iso-dense, low-density, or mixed density shadows beneath the inner table of the skull and on the brain surface seen on cranial CT imaging); (3) outcome measures: main outcome measures included a) surgical success rate: many studies had different definitions for "success" and lacked long-term follow-up results. We defined surgical success as follows: the clinical symptoms were almost or complete disappeared with the imageology showed that the hematoma was removed obviously (the volume was lessen over 50\%); b) a recurrence rate of postoperative hematoma; and c) postoperative complications. Secondary outcome measures included acute postoperative bleeding, mortality, length of hospital stay, and hospital costs; (4) the languages were restricted to Chinese and English.

Exclusion criteria included: (1) studies with only an abstract and lacking a full text with no response from the authors when contacted; (2) studies with incomplete data or the data that could not be calculated from the original study and that were not described in detail; (3) two studies with similar results from the same institution; (4) subjects who suffered from other diseases besides CSDH, such as cerebral hemorrhage, epidural hematoma, severe systemic disease, severe liver and kidney dysfunction, acute hemorrhage, and brain hernia; (5) subjects with a history of intracranial hemorrhage, infection, tumors, and craniotomy.

\section{Data Extraction and Quality Appraisal}

Two investigators independently screened the studies and completed the data extraction by screening the title, abstract, and full text in strict accordance with the pre-defined inclusion and exclusion criteria. The extracted contents included the first author, publication time, sample size, baseline conditions, intervention, and outcome measures. Two investigators then cross-checked the results, and the studies with conflicting opinions were decided upon after discussion by the research team. The quality of randomized controlled trials was appraised using the Cochrane systematic review criteria, and the quality of non-randomized case-control studies was appraised using the Newcastle-Ottawa scoring system (NOS; including selection, comparability, and exposure or outcome, and the highest quality studies are awarded up to nine stars). 


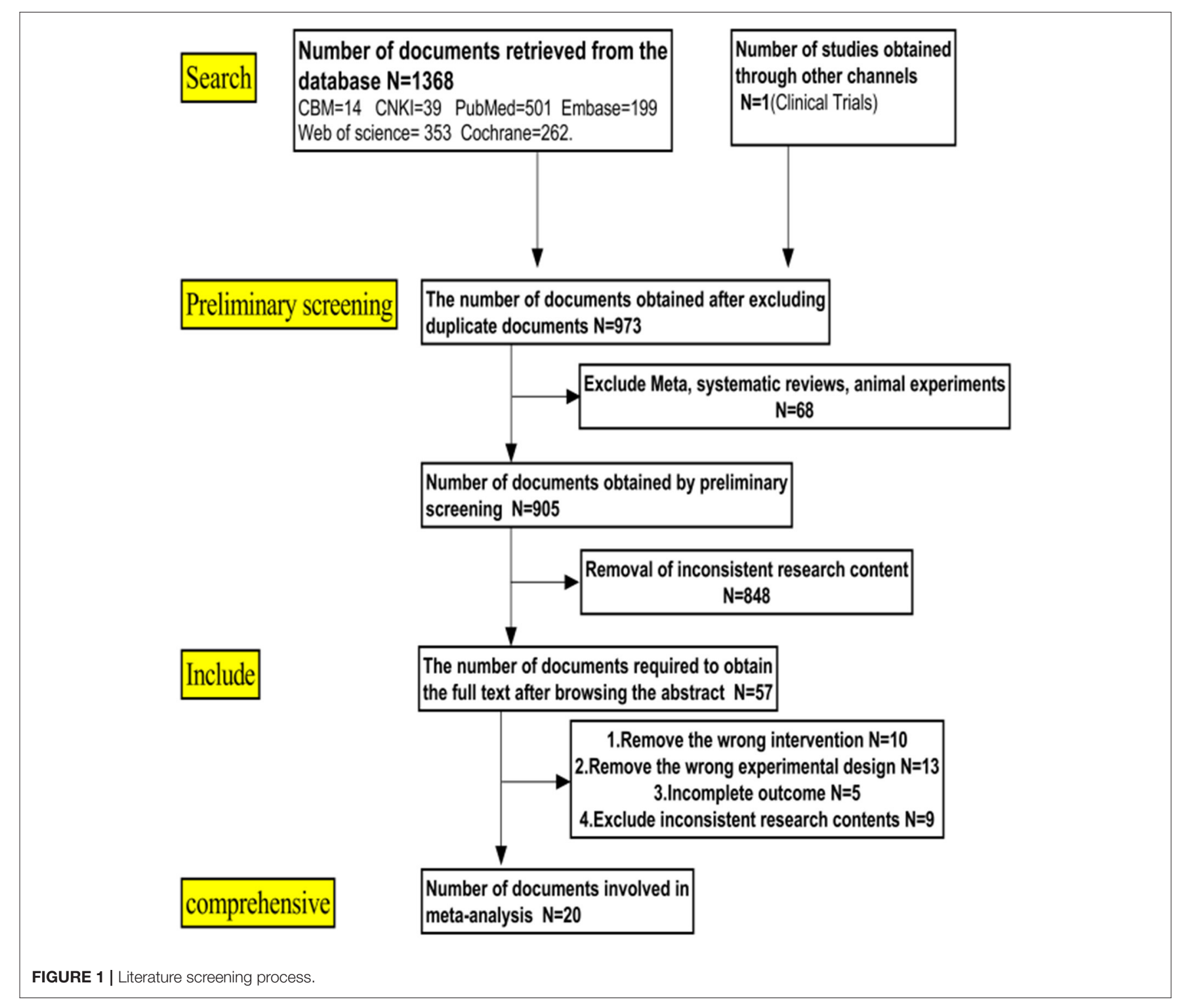

\section{Statistical Analysis}

Meta-analysis was performed using RevMan 5.3 software or STATA provided by the Cochrane Collaboration. Relative risk (RR) was used for data enumeration and standardized mean difference (WMD) was used for data measurement, and $95 \%$ confidence interval was also calculated. The heterogeneity of the included studies was tested, and the $\chi^{2}$-test was employed to analyze the statistical heterogeneity. If $I^{2} \leq 50 \%(P<$ 0.1 ), it indicated that the heterogeneity was small, and a fixed-effect model was used for meta-analysis. If $I^{2}>50 \%$ $(P>0.1)$, it indicated that the heterogeneity was significant, and the sources of heterogeneity were analyzed by L' Abbe plot, radial plot, sensitivity analysis, meta-regression, and clinical analysis. Furthermore, subgroup analysis was performed when necessary. If no clinical heterogeneity was present, a random-effect model was used for meta-analysis. Funnel plots and Egger's test were also used to analyze potential publication bias.

\section{RESULTS}

\section{Search Results}

A total of 1,369 studies were obtained in the initial search, and 20 studies (13-32) were finally included based on the inclusion and exclusion criteria. These 20 studies included 17 English articles (13-20, 24-32) and 3 Chinese articles (2123), of which four of them were randomized controlled trials $(15,17,20,21)$ while the remaining 16 were case-control trials. The workflow of literature retrieval and screening is shown in Figure 1. 
TABLE 1 | Basic characteristics of included studies.

\begin{tabular}{|c|c|c|c|c|c|c|c|c|c|c|c|c|}
\hline \multirow[t]{3}{*}{ References } & \multicolumn{2}{|c|}{ Number of cases } & \multicolumn{2}{|c|}{ Gender (male/female) } & \multicolumn{2}{|c|}{ Age (years) } & \multicolumn{2}{|c|}{ Intervention } & \multicolumn{2}{|c|}{ Follow-up } & \multicolumn{2}{|c|}{ Hospital costs } \\
\hline & & & & & & & & & & & $\mathbf{T}$ & C \\
\hline & $\mathbf{T}$ & C & $\mathbf{T}$ & C & $\mathbf{T}$ & C & $\mathbf{T}$ & C & $\mathbf{T}$ & C & $108391 \pm 11124(\$)$ & $166318 \pm 21452$ (\$) \\
\hline Golub et al. (13) & 39 & 68 & $29 / 10$ & $48 / 22$ & $68.7 \pm 2.4$ & $66.8 \pm 2.2$ & S & $\mathrm{B}$ & \multicolumn{2}{|c|}{$3 \mathrm{~W}$} & \multicolumn{2}{|r|}{ NA } \\
\hline Muzii et al. (15) & 22 & 24 & $14 / 8$ & $16 / 8$ & 78.7 & 76.3 & $\mathrm{H}$ & B & \multicolumn{2}{|c|}{$2 \mathrm{M}$} & \multicolumn{2}{|r|}{ NA } \\
\hline Rughani et al. (16) & 21 & 21 & $14 / 7$ & $14 / 7$ & 73 & 73.3 & S & B & $66.8 \mathrm{D}$ & $45 \mathrm{D}$ & & NA \\
\hline Singh et al. (17) & 48 & 52 & $43 / 5$ & $47 / 5$ & 59.8 & 61.2 & $\mathrm{H}$ & B & \multicolumn{2}{|c|}{$3 \mathrm{M}$} & \multicolumn{2}{|r|}{ NA } \\
\hline Szmuda et al. (18) & 5 & 27 & $3 / 2$ & $20 / 7$ & 69 & 72.2 & $\mathrm{H}$ & B & \multicolumn{2}{|c|}{ NA } & $8685.98 \pm 1531.42$ & $11759.85 \pm 2363.21(¥)$ \\
\hline Wang et al. (19) & 68 & 53 & $57 / 11$ & $44 / 9$ & $69.49 \pm 12.69$ & $66.67 \pm 13.13$ & Y & B & \multicolumn{2}{|c|}{$1 \mathrm{M}$} & \multirow{2}{*}{\multicolumn{2}{|c|}{$\begin{array}{l}\text { NA } \\
\text { NA }\end{array}$}} \\
\hline Xu et al. (20) & 20 & 20 & $16 / 4$ & $17 / 3$ & $66.20 \pm 10.11$ & $66.00 \pm 16.74$ & Y & B & \multicolumn{2}{|c|}{$3 \mathrm{M}$} & & \\
\hline Teng et al. (21) & 30 & 30 & $16 / 14$ & $17 / 13$ & $62.1 \pm 0.47$ & $62.09 \pm 0.48$ & Y & B & \multicolumn{2}{|c|}{$1 \mathrm{M}$} & & NA \\
\hline Wang et al. (19) & 28 & 38 & NA & $68.2 \pm 18.5$ & $67.3 \pm 12.9$ & $\mathrm{H}$ & B & & \multicolumn{2}{|c|}{$3 \mathrm{M}$} & & NA \\
\hline Liu et al. (22) & 17 & 44 & $16 / 1$ & $41 / 3$ & 57 & 60 & Y & B & \multicolumn{2}{|c|}{$3 \mathrm{M}$} & & NA \\
\hline Zhu et al. (23) & 66 & 69 & $55 / 11$ & $60 / 9$ & 65 & 61 & Y & B & \multicolumn{2}{|c|}{$3 \mathrm{M}$} & & NA \\
\hline Flint et al. (24) & 371 & 659 & $257 / 117$ & 464/195 & 75 & 76 & S & $\mathrm{B}$ & \multicolumn{2}{|c|}{$6 \mathrm{M}$} & & NA \\
\hline Ortiz et al. (25) & 25 & 41 & NA & 71.5 & 71.3 & $S$ & B & & \multicolumn{2}{|c|}{ NA } & & NA \\
\hline Xu et al. (26) & 116 & 42 & $101 / 15$ & $33 / 9$ & $65.3 \pm 14.2$ & $67.5 \pm 14.56$ & Y & B & \multicolumn{2}{|c|}{$3 \mathrm{M}$} & & NA \\
\hline Safain et al. (27) & 23 & 23 & $15 / 8$ & $14 / 9$ & 68 & 61 & $\mathrm{H}$ & $\mathrm{B}$ & $12 \mathrm{~W}$ & $15 \mathrm{~W}$ & & NA \\
\hline Smely et al. (28) & 33 & 33 & $21 / 12$ & $21 / 12$ & 69.7 & 70 & $\mathrm{H}$ & $\mathrm{B}$ & 81D & $82 \mathrm{D}$ & & NA \\
\hline Gabarros et al. (29) & 105 & 83 & NA & NA & S & $\mathrm{B}$ & & & $N$ & & & \\
\hline Balser et al. (30) & 29 & 44 & $29 / 0$ & $44 / 0$ & 76.6 & 78.4 & $\mathrm{H}$ & $\mathrm{B}$ & 3 & & $48446 \pm 33226(\$)$ & $67227 \pm 6457(\$)$ \\
\hline Wan et al. (31) & 31 & 31 & $19 / 12$ & $18 / 13$ & 72.5 & 73.6 & Y & B & 31 & & & NA \\
\hline Fei et al. (32) & 17 & 19 & $11 / 6$ & $15 / 4$ & 85 & 75 & Y & $\mathrm{B}$ & 6 & & & NA \\
\hline
\end{tabular}

$S$, the Subdural Evacuating Port System; B, burr hole craniotomy; $Y, Y L-1$ needle; $D$, day; $W$, week; $M$, month; y, year. 


\section{Characteristics of the Included Studies}

Twenty studies with a total of 2,536 cases were included, including 1,114 cases in the experimental group and 1,422 in the control group. The baseline of patients was comparable. All experimental groups of the included studies were treated with TDC with hollow screws (SEPS, the hollow screw, or YL1 needle) for $\mathrm{CSDH}$, while the control groups were treated with BHC. The basic characteristics of the included studies are listed in Table 1.

\section{Quality Appraisal}

Four randomized controlled studies reported random allocation methods with complete results data, no selective report, no conflict of interest, and a balanced baseline. Only two studies reflected the allocation concealment $(15,20)$, and similarly, only two articles reported the implementation of blinding $(17,20)$ (Figure 2). All 16 case-control trials were scored using the NOS scoring system. Eight studies were scored as eight stars, three were scored as seven stars, three were scored as six stars, and two articles were scored as five stars (Table 2).

\section{Meta-Analysis Results}

1) Surgical success rate: a total of 20 studies mentioned the surgical success rate, and there was no statistical heterogeneity among the studies $\left(I^{2}=21 \%<50 \%, P=0.20>\right.$ 0.1 ). The fixed-effect model was employed for effect size combination. The results showed that the surgical success rate of TDC with hollow screws was 1.03 times that of BHC, but there was no statistical significance $(\mathrm{RR}=1.03, P=0.05$; Figure 3). Since the type of puncture needle could affect the combined outcome measure (success rate), subgroup analysis was performed.

Subgroup analysis: The puncture needle used in 8 of 20 included studies was the YL-1 needle, and there was no statistical heterogeneity among the eight studies $\left(I^{2}=31 \%<50 \%, P=\right.$ 0.18). A fixed-effect model was chosen for combination, and the results showed that the surgical success rate of the YL-1 needle was 1.06 times that of $\mathrm{BHC}$, with statistical significance $(\mathrm{RR}=$ $1.05, P=0.02$; Figure 4).

2) Postoperative recurrence rate: $A$ total of 20 studies reported postoperative recurrence rate, and there was statistical heterogeneity among the studies $\left(I^{2}=51 \%>50 \%, P=0.005\right.$ $<0.1)$. A random-effect model was employed for combination. Results: The recurrence rate of TDC with hollow screws was 1.13 times that of $\mathrm{BHC}$, with no statistical significance $(\mathrm{RR}=1.13$, $P=0.50$; Figure 5). There was mild to moderate heterogeneity among the included studies in this review. In addition to using a random-effect combination, it was also necessary to explore the root of heterogeneity.

Sensitivity analysis: The results of the L'Abbe and radial plots suggested that some studies had the possibility for heterogeneity (Figure 6). The sensitivity analysis did not find studies with a significant effect on heterogeneity (Figure 6). Subsequently, meta-regression was conducted, where the patients were divided into the YL-1 needle group and other puncture needle groups based on the intervention measures of the

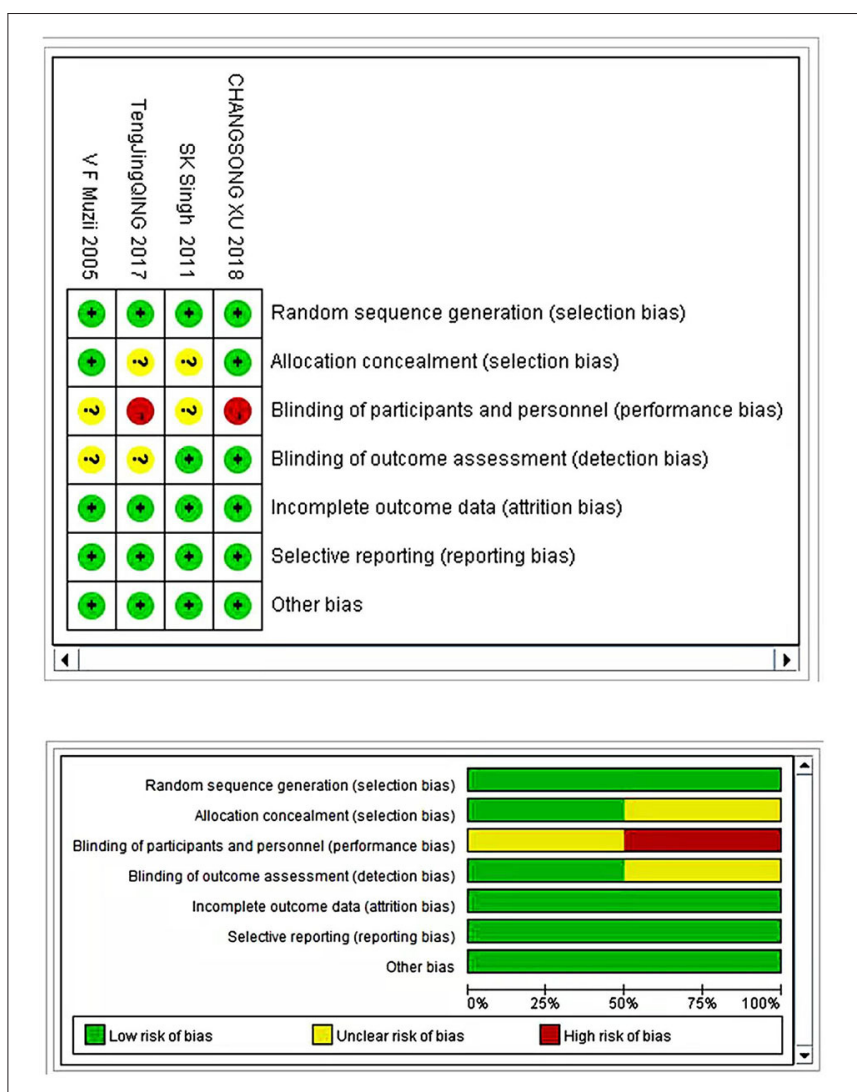

FIGURE 2 | Cochrane assessment result for risk of bias.

covariates. The results showed that the control measures were the source of heterogeneity (Figure 6). Therefore, subgroup analysis was performed.

Subgroup analysis: There was no statistical heterogeneity among the studies in the YL- 1 needle group $\left(I^{2}=41 \%<50 \%\right.$, $P=0.105>0.1)$ and in other puncture needle groups $\left(I^{2}=32 \%\right.$ $<50 \%, P=0.135)$. A fixed-effect model was used.

Results: The recurrence rate of the YL-1 needle group was $50.3 \%$ that of $\mathrm{BHC}$, with statistical significance $(\mathrm{RR}=0.503, \mathrm{P}$ $=0.002$; Figure 7 ). The recurrence rate of other puncture needle groups was 1.483 times that of $\mathrm{BHC}$, with statistical significance $(\mathrm{RR}=1.483, P=0.001$; Figure 7$)$.

3) Postoperative complications: A total of 19 included studies mentioned postoperative complications, without statistical heterogeneity among the studies $\left(I^{2}=20 \%<50 \%\right.$, $P=0.21)$. A fixed-effect model was used. Results of metaanalysis: the postoperative complications of TDC with hollow screws were $57 \%$ that of the BHC group, which was much lower, with statistical significance $(\mathrm{RR}=0.57, P=0.0002$; Supplementary Materials).

4) Acute intracranial hemorrhage: A total of 13 studies contained relevant data, and there was no statistical heterogeneity among the studies $\left(I^{2}=0.0 \%<50 \%, P=0.861\right)$. Using the fixed-effect model, the results showed that the acute intracranial 
TABLE 2 | Quality assessment of NOS.

\begin{tabular}{|c|c|c|c|c|}
\hline References & Selection & Comparability & Outcome & Total \\
\hline Golub et al. (13) & $\star \star \star$ & $\star \star$ & $\star \star \star$ & 8 \\
\hline Wang et al. (14) & $\star \star \star$ & $\star \star$ & $\star \star \star$ & 8 \\
\hline Rughani et al. (16) & $\star \star \star$ & $\star \star \star$ & $\star \star \star$ & 8 \\
\hline Szmuda et al. (18) & $\star \star$ & & $\star \star \star$ & 5 \\
\hline Wang et al. (19) & $\star \star \star$ & $\star \star$ & $\star \star \star$ & 8 \\
\hline Liu et al. (22) & $\star \star$ & & $\star \star \star$ & 5 \\
\hline Zhu et al. (23) & $\star \star \star$ & & $\star \star \star$ & 6 \\
\hline Flint et al. (24) & $\star \star \star$ & $\star \star$ & $\star \star \star$ & 8 \\
\hline Ortiz et al. (25) & $\star \star \star$ & & $\star \star \star$ & 6 \\
\hline Xu et al. (26) & $\star \star$ & $\star \star$ & $\star \star \star$ & 7 \\
\hline Safain et al. (27) & $\star \star \star$ & & $\star \star \star$ & 6 \\
\hline Smely et al. (28) & $\star \star \star$ & $\star \star$ & $\star \star \star$ & 8 \\
\hline Gabarros et al. (29) & $\star \star \star$ & $\star \star$ & $\star \star \star$ & 8 \\
\hline Balser et al. (30) & $\star \star \star$ & $\star$ & $\star \star \star$ & 7 \\
\hline Wan et al. (31) & $\star \star \star$ & $\star \star \star$ & $\star \star \star$ & 8 \\
\hline Fei et al. (32) & $\star \star \star$ & $\star$ & $\star \star \star \star$ & 7 \\
\hline
\end{tabular}

NOS used the semi-quantitative principle of star system to evaluate the quality of literature, with a full score of 9 " $\star$, $\star \star, ~ \star \star \star$ ".

hemorrhage rate after TDC with hollow screws was $58.4 \%$ that of $\mathrm{BHC}$, which was much lower, with statistical significance $(\mathrm{RR}=$ $0.584, P=0.027$; Supplementary Materials).

5) Mortality: A total of 18 studies reported mortality, and there was no statistical heterogeneity among the studies $\left(I^{2}=0 \%<\right.$ $50 \%, P=0.68>0.1)$. The fixed-effect model was used. Results: the mortality of TDC with hollow screws was slightly higher than that of $\mathrm{BHC}$, but there was no statistical significance $(\mathrm{OR}=1.01$, $\mathrm{P}=0.95>0.05$; Supplementary Materials). Heterogeneity and meta-analysis were performed for YL-1 needle groups, and the results were similar (Supplementary Materials).

6) Length of hospital stay: A total of eight studies were included, and there was statistical heterogeneity among the studies $\left(I^{2}=99.2 \%>50 \%, P<0.001\right)$. The random-effect model was used. The results showed that the hospital stay for TDC with hollow screws was significantly shorter by 3.752 days, with statistical significance $(\mathrm{WMD}=-3.752, P<0.001$; Supplementary Materials).

Sensitivity analysis and meta-regression: No study with a significant effect on heterogeneity was found in the sensitivity analysis (Supplementary Materials); therefore, the patients were divided into YL-1 needle and other puncture needle groups according to the intervention measure. No obvious source of heterogeneity was found in the meta-regression results (Supplementary Materials). Considering that the type of puncture needle could affect the combined outcome measure (length of hospital stay), subgroup analysis was performed.

Subgroup analysis: The intervention measure with YL-1 needles was used by six studies, and these studies had statistical heterogeneity $\left(I^{2}=96.6 \%>50 \%, P=0.000<0.1\right)$. The randomeffect model was used. Results: the length of hospital stay in the YL-1 needle group was more than 5 days shorter than that of the BHC group, with statistical significance $(\mathrm{WMD}=-5.032, P$
$<0.001$; Supplementary Materials). The other two studies had no statistical heterogeneity $\left(I^{2}=25 \%<50 \%, P=0.248\right)$, and the results using fixed-effect model showed that the length of hospital stay in the other puncture needle group was 1.804 days less than that of the $\mathrm{BHC}$ group $(\mathrm{WMD}=-1.804, P<0.001$; Supplementary Materials).

7) Bias test: The funnel plot and Egger's test were plotted for the surgical success rate, recurrence rate, complications, acute intracranial hemorrhage, mortality, hospital stay, and subgroup analyses. It was found that only the surgical success rate had publication bias ( $P=0.019$, Supplementary Materials). Trimand-fill method was used for correction. After four iterations, the results of three studies were simulated. The effect size of 23 studies after combination was 1.019, which was not significantly reversed compared with the results before the trim-and-fill, and the results of the meta-analysis were stable (Supplementary Materials). The remaining studies had no publication bias (Supplementary Materials).

\section{DISCUSSION}

Patients with $\mathrm{CSDH}$ having definite clinical symptoms, large hematoma volume, and no surgical contraindications need to undergo surgical treatment. Traditional neurosurgical modalities include craniostomy hematoma evacuation, BHC in the operating room, and TDC. Hematoma evacuation using craniotomy is no longer a primary option in clinical practice due to its high mortality rate (as high as 29\%) (33). It is only considered in a few patients with intractable $\mathrm{CSDH}$ or severe capsular thickening and calcification. With further study of the pathogenesis of $\mathrm{CSDH}, \mathrm{McKissock}$ et al. successfully cured patients with $\mathrm{CSDH}$ using $\mathrm{BHC}$ in the operating room for the 


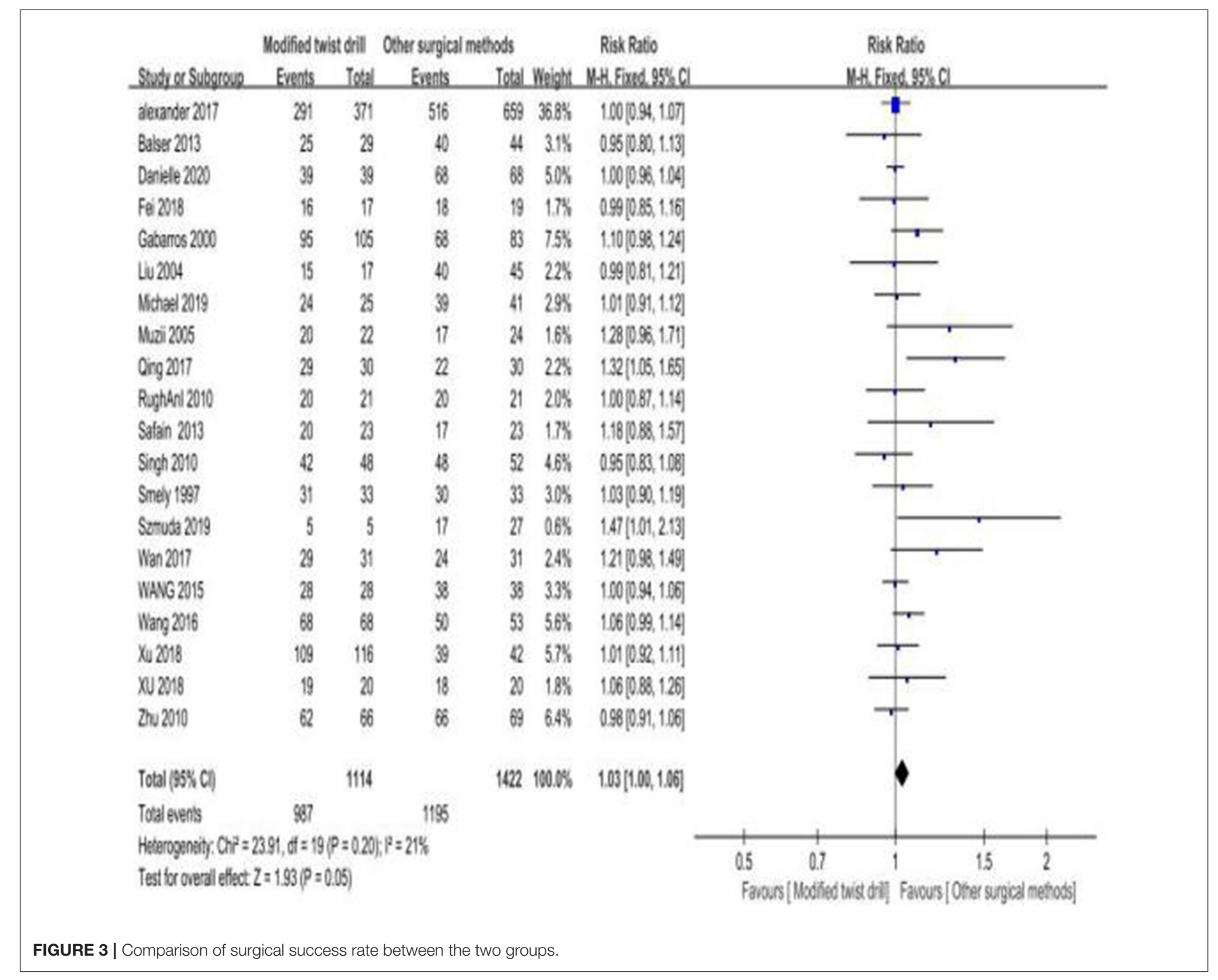

first time. Thus, BHC became the first choice for the treatment of $\mathrm{CSDH}$ in clinical practice because of its simple operation, high success rate, and relatively better mortality rate (34). However, it also possesses several notable disadvantages in clinical practice, which include general anesthesia, large operating area, poor tolerance in elderly patients, acute intracranial hemorrhage, and high postoperative recurrence rate. Recently, TDC with hollow screws (e.g., SEPS, the hollow screw, and YL-1 needle), which is a bedside procedure, has been favored by frontline physicians. Although many studies compared the efficacy and safety of TDC with hollow screws and BHC in the treatment of CSDH, the results were not consistent $(9,10,12,16)$. Additionally, although there are evidence-based studies on minimally invasive punctures, they are either old or do not consider the different needle types. Hence, this review comprehensively assessed the efficacy and safety of the two groups by combining and comparing the relevant data between the two groups of TDC with hollow screws and BHC.
The surgical success rate is a significant indicator for evaluating the efficacy of surgery. This review indicated that the success rate of TDC with hollow screws was slightly higher than that of the BHC group, but the difference was not statistically significant, which is also in line with most results of previous studies. The results of subgroup analysis suggested that the surgical success rate of the YL-1 needle was slightly higher than that of the BHC group with statistical significance.

The recurrence of hematoma is also a difficult problem in the treatment of $\mathrm{CSDH}$. Reducing the recurrence is equal to reducing the complications and medical costs. The results of this review showed that the recurrence rate of TDC with hollow screws was similar to that of the $\mathrm{BHC}$ group. The results of subgroup analysis suggested that the recurrence rate of the YL-1 needle was only about half that of the $\mathrm{BHC}$ group. Postoperative complications of CSDH are an important cause of postoperative reduced quality of life. We first analyzed the total complication 


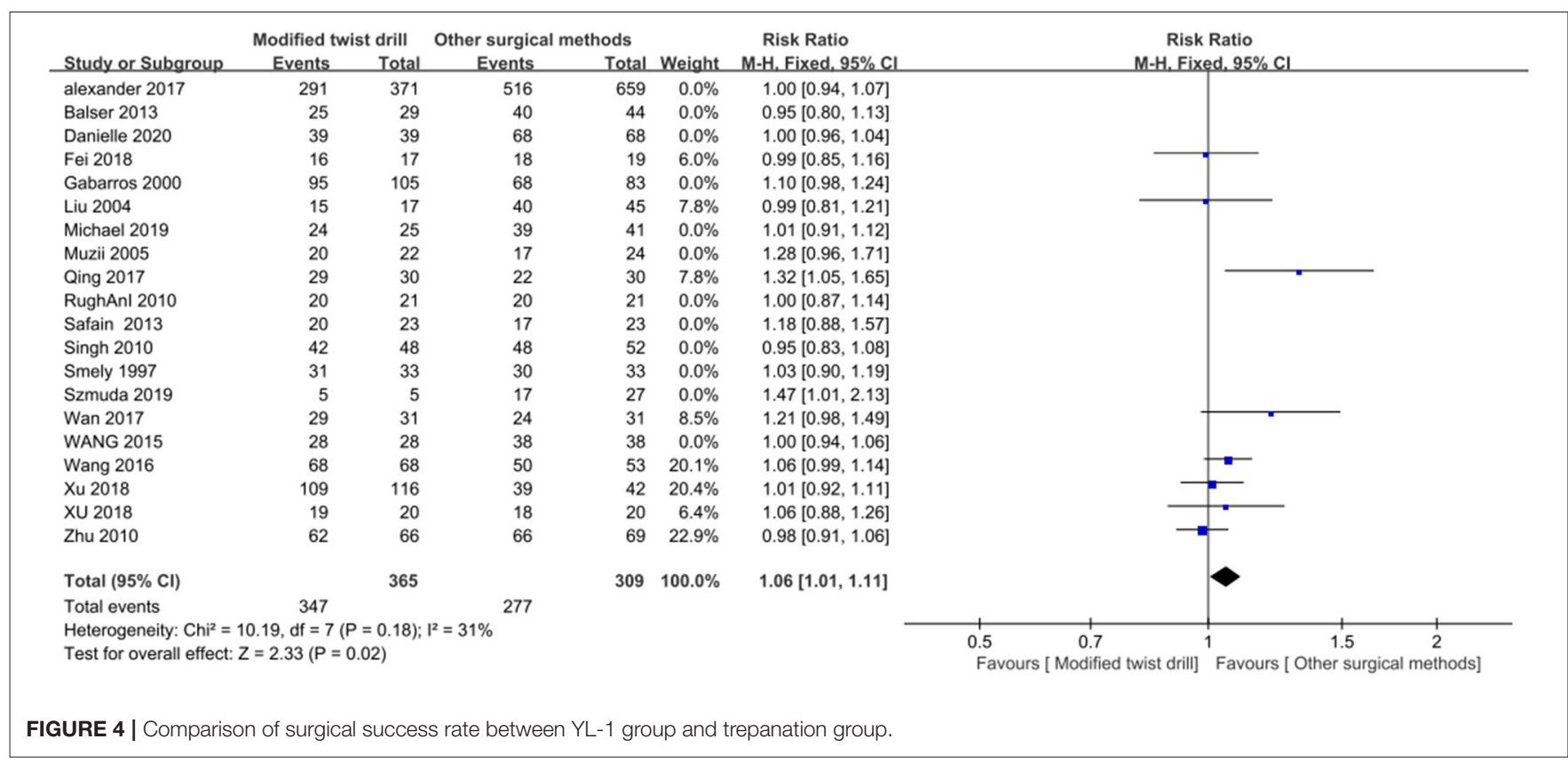

\begin{tabular}{|c|c|c|c|c|c|c|c|c|c|c|}
\hline \multirow[b]{2}{*}{ Study or Subgroup } & \multicolumn{2}{|c|}{ Modified twist drill } & \multicolumn{2}{|c|}{ Other surgical methods } & \multirow{2}{*}{ Weight } & \multirow{2}{*}{$\begin{array}{l}\text { Risk Ratio } \\
\text { M-H. Random, } 25 \% \mathrm{Cl}\end{array}$} & \multirow{2}{*}{\multicolumn{4}{|c|}{$\begin{array}{l}\text { Risk Ratio } \\
\text { M-H. Random. } 95 \% \mathrm{CI}\end{array}$}} \\
\hline & Events & Total & Events & Total & & & & & & \\
\hline alexander 2017 & 50 & 371 & 60 & 659 & $11.1 \%$ & $1.48[1.04,2.11]$ & & & $\rightarrow$ & \\
\hline Balser 2013 & 4 & 29 & 4 & 44 & $4.8 \%$ & $1.52[0.41,5.59]$ & & & 5 & \\
\hline Danielle 2020 & 13 & 39 & 9 & 68 & $8.1 \%$ & $2.52[1.19,5.35]$ & & & $\longrightarrow$ & \\
\hline Fei 2018 & 1 & 17 & 1 & 19 & $1.6 \%$ & $1.12[0.08,16.52]$ & & & & \\
\hline Gabarros 2000 & 14 & 105 & 9 & 83 & $7.9 \%$ & $1.23[0.56,2.70]$ & & - & - & \\
\hline Liu 2004 & 5 & 17 & 8 & 45 & $6.6 \%$ & $1.65[0.63,4.36]$ & & & $\cdot-$ & \\
\hline Michael 2019 & 10 & 25 & 7 & 41 & $7.6 \%$ & $2.34[1.02,5.36]$ & & & ? & \\
\hline Muzii 2005 & 1 & 22 & 5 & 24 & $2.5 \%$ & $0.22[0.03,1.72]$ & & & - & \\
\hline Qing 2017 & 1 & 30 & 2 & 30 & $2.0 \%$ & $0.50[0.05,5.22]$ & & & & \\
\hline RughAnl 2010 & 5 & 21 & 4 & 21 & $5.5 \%$ & $1.25[0.39,4.02]$ & & & 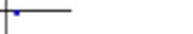 & \\
\hline Safain 2013 & 4 & 23 & 2 & 23 & $3.7 \%$ & $2.00[0.41,9.87]$ & & & & \\
\hline Singh 2010 & 4 & 48 & 1 & 52 & $2.3 \%$ & $4.33[0.50,37.42]$ & & & & \\
\hline Smely 1997 & 6 & 33 & 11 & 33 & $7.3 \%$ & $0.55[0.23,1.30]$ & & & - & \\
\hline Szmuda 2019 & 2 & 5 & 1 & 27 & $2.3 \%$ & $10.80[1.19,97.73]$ & & & & \\
\hline Wan 2017 & 1 & 31 & 13 & 31 & $2.7 \%$ & $0.08[0.01,0.55]$ & & & & \\
\hline WANG 2015 & 7 & 28 & 5 & 38 & $6.2 \%$ & $1.90[0.67,5.37]$ & & & & \\
\hline Wang 2016 & 9 & 68 & 9 & 53 & $7.4 \%$ & $0.78[0.33,1.83]$ & & - & — & \\
\hline Xu 2018 & 8 & 116 & 8 & 42 & $7.0 \%$ & $0.36[0.15,0.90]$ & & & & \\
\hline XU 2018 & 0 & 20 & 3 & 20 & $1.4 \%$ & $0.14[0.01,2.60]$ & & & & \\
\hline Zhu 2010 & 1 & 66 & 2 & 69 & $2.0 \%$ & $0.52[0.05,5.63]$ & & & & \\
\hline Total $(95 \% \mathrm{Cl})$ & & 1114 & & 1422 & $100.0 \%$ & $1.13[0.79,1.63]$ & & & & \\
\hline \multirow{2}{*}{\multicolumn{6}{|c|}{ Heterogeneity: $\operatorname{Tau}^{2}=0.28 ; \mathrm{Chi}^{2}=38.79, \mathrm{df}=19(\mathrm{P}=0.005) ; \mathrm{I}^{2}=51 \%$}} & & & & & \\
\hline & & & & & & & 0.005 & 0.11 & 110 & 200 \\
\hline
\end{tabular}

rate. The results showed that the rate of TDC with hollow screws was much lower than that of BHC, approximately half that of the BHC group. Common postoperative complications of $\mathrm{CSDH}$ include intracranial hemorrhage, seizures, and infection. Seizures are the most common complication of CSDH. As with other factors stimulating the cortex, patients could develop seizures due to the presence of a hematoma and possible changes in the amount of hematoma (35). Therefore, many surgeons use antiepileptic drugs in the perioperative period to reduce the risk of seizures due to changes in intracranial pressure, traumatic brain injury, and possible postoperative bleeding based on clinical experience. Multivariate logistic regression analysis by Grobelny et al. (36) also demonstrated that preoperative antiepileptic drugs were the only factor 

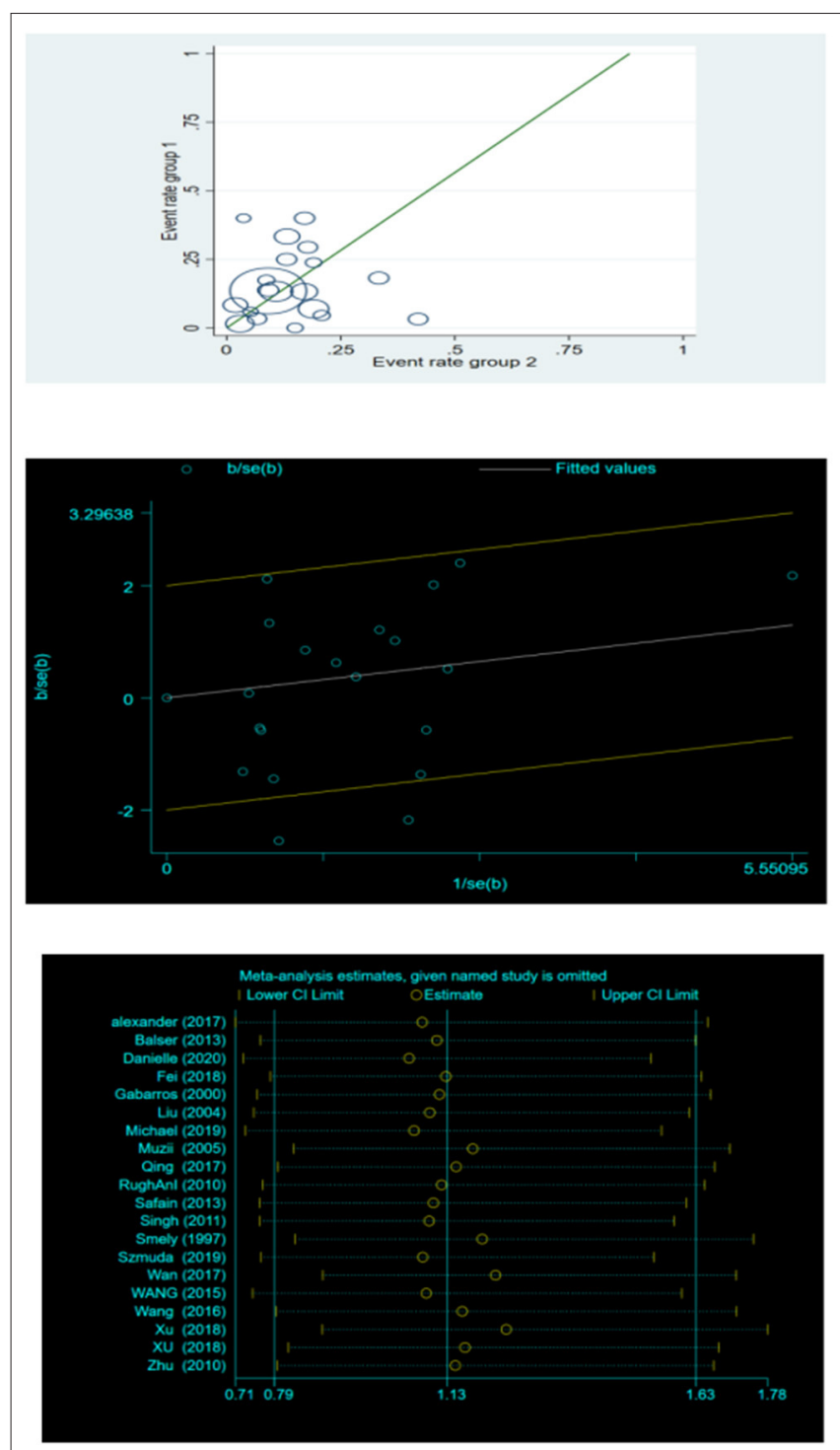

\section{Meta-regression}

REML estimate of between-study variance

$\%$ residual variation due to heterogeneity

Proportion of between-study variance explained With Knapp-Hartung modification

\begin{tabular}{r|rrrrrr}
\hline logRR & Coef. & Std. Err. & $t$ & $p\rangle|t|$ & [95\% Conf. Interval] \\
\hline intervention & -.9596829 & .366725 & -2.62 & 0.017 & -1.730144 & -.1892223 \\
cons & .4291487 & .1963271 & 2.19 & 0.042 & .0166808 & .8416167 \\
\hline
\end{tabular}

FIGURE 6 | L'Abbe plot, radial plot, sensitivity analysis and meta regression for postoperative recurrence rate.

responsible for postoperative seizure reduction. Due to the above clinical experience and related studies, many medical institutions routinely use antiepileptic drugs to prevent seizures after surgery, and many studies lack the record of antiepileptic dose and duration. Since antiepileptic drugs prevent seizures, we did not include seizures as an outcome measure. As frontline neurosurgeons in clinical practice, we were more worried about acute postoperative intracranial hemorrhage, which is an important cause of poor surgical results. The results of this review showed that the rate of acute intracranial hemorrhage was much lower in TDC with hollow screws. Moreover, the mortality of TDC with hollow screws group was also lower than that of the BHC group.

The results of this review indicated that the length of hospital stay was significantly lower for TDC with hollow screws than for BHC. The subgroup analysis showed that the length of hospital stay was significantly shorter in the YL-1 needle group than in the BHC group. Combined with the findings above and surgical experience, TDC with hollow screws has the following advantages: 1 ) The puncture needle that integrates the drilling can directly reach the hematoma with less bleeding and injury after accurate preoperative positioning, thus reducing the patient's pain; 2) TDC with hollow screws is easy to operate, and it can be implemented at the bedside with local anesthesia, thereby avoiding general anesthesia and shortening the length of hospital stay; 3) The puncture needle is short and penetrates the dural layer, which avoids brain tissue injury. In addition, the puncture needle has good airtight drainage, which reduces the incidence of intracranial infection and pneumocephalus, thus reducing complications; 4) There are no special surgical contraindications, and it is suitable for $\mathrm{CSDH}$ patients that are older or in poor conditions; 5) The surgical equipment is simple and cheap; 6) Although the puncture needle has a small aperture and is easily blocked by blood clots resulting in incomplete drainage, the closed drainage system of the YL-1 needle contains a three-way valve that can be injected with urokinase to dissolve the blood clot after surgery. Altogether, we found that the above might contribute to its lower recurrence rate.

The limitations of this review were as follows:

1) Although several cases of the case-control studies met the inclusion criteria, the intervention group was quite small.

2) While extensive searches were carried out in many databases, we eventually only included studies in Chinese and English, which could lead to selection bias. In addition, three Chinese studies were included, which could have led to a regional deviation in the final conclusion.

3) When the hospitalization time was combined, there was great heterogeneity, and the significant heterogeneity could be derived from clinical heterogeneity, including the patient population (e.g., age, education level, and underlying diseases), the size and location of the initial hematoma, different perioperative management, and the operation proficiency of surgeons, and the inconsistent discharge criteria in each medical institution.

\section{CONCLUSION}

In summary, TDC with hollow screws is a relatively effective first-line treatment for CSDH. TDC with hollow screws has 

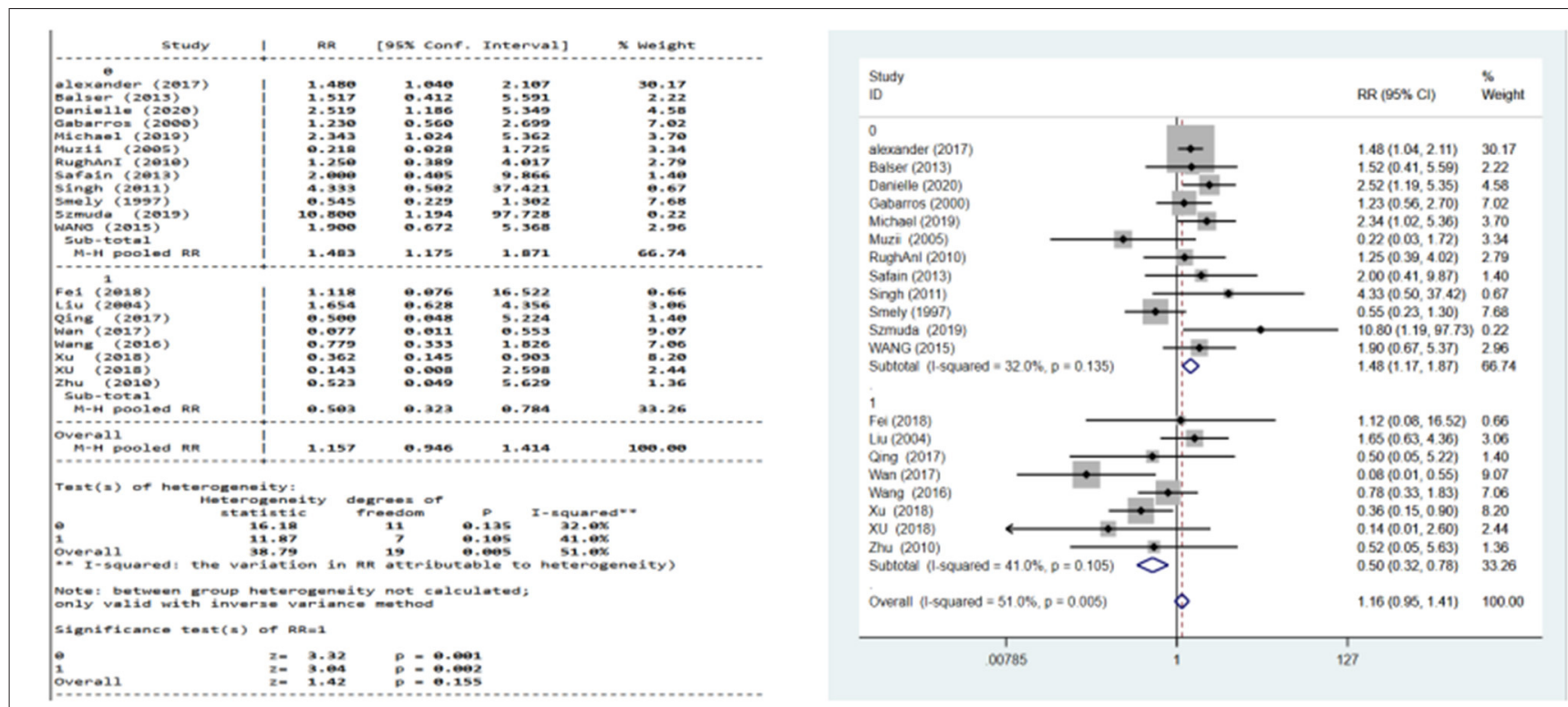

FIGURE 7 | Subgroup analysis for postoperative recurrence rate.

a lower incidence of acute intracranial hemorrhage, overall complication rate, and length of hospital stay. Nevertheless, further confirmation using a larger sample of multicenter, highquality clinical randomized controlled trial is warranted.

\section{DATA AVAILABILITY STATEMENT}

The original contributions presented in the study are included in the article/Supplementary Material, further inquiries can be directed to the corresponding author.

\section{AUTHOR CONTRIBUTIONS}

ZW: conception and design and manuscript writing. CW: administrative support. ZW and HJ: provision of study materials

\section{REFERENCES}

1. Karibe H, Kameyama M, Kawase M, Hirano T, Kawaguchi T, Tominaga T. Epidemiology of chronic subdural hematomas. No Shinkei Geka. (2011) 39:1149-53.

2. Foelholm R, Waltimo O. Epidemiology of chronic subdural haematoma. Acta Neurochir (Wien). (1975) 32:247-50. doi: 10.1007/BF0140 5457

3. Kudo H, Kuwamura K, Izawa I, Sawa H, Tamaki N. Chronic subdural hematoma in elderly people: present statuson Awaji Island and epidemiological prospect. Neurol Med Chir (Tokyo). (1992) 32:207-9. doi: 10.2176/nmc.32.207

4. Asghar M, Adhiyaman V, Greenway MW, Bhowmick BK, Bates A. Chronic subdural haematoma in the elderly-a North Wales experience. J R Soc Med. (2002) 95:290-2. doi: 10.1258/jrsm.95.6.290

5. Balser D, Farooq S, Mehmood T, Reyes M,Samadani M. Actual andprojected incidence rates for chronic subdural hematomas in United States veterans or patients. ZW, YW, and HJ: collection and assembly of data. ZW and YW: data analysis and interpretation. All authors final approval of manuscript.

\section{FUNDING}

This work was supported by Doctor Startup Fund (No. BSQDJ0130). PROSPERO Registration Number: CRD42021270835.

\section{SUPPLEMENTARY MATERIAL}

The Supplementary Material for this article can be found online at: https://www.frontiersin.org/articles/10.3389/fneur. 2021.811873/full\#supplementary-material

administrationand civilian populations. J Neurosurg. (2015) 123:120915. doi: $10.3171 / 2014$.9.JNS141550

6. Yang W, Huang J. Chronic subdural hematoma: epidemiology and natural history. Neurosurg Clin N Am. (2017) 28:20510. doi: 10.1016/j.nec.2016.11.002

7. Ivamoto HS, Lemos HP, Atallah AN. Surgical treatments for chronic subdural hematomas: a comprehensive systematic review. World Neurosurg. (2016) 86:399-418. doi: 10.1016/j.wneu.2015.10.025

8. Kolias AG, Chari A, Santarius T, Hutchinson PJ. Chronic subdural haematoma: modern management andemerging therapies. Nat Rev Neurol. (2014) 10:570-8. doi: 10.1038/nrneurol.2014.163

9. Weigel R, Schmiedek P, Krauss JK. Outcome of contemporary surgery for chronic subdural haematoma: evidence based review. J Neurol Neurosurg Psychiatry. (2003) 74:937-43. doi: 10.1136/jnnp.74.7.937

10. Lega BC, Danish SF, Malhotra NR, Sonnad SS, Stein SC. Choosing the best operation for chronic subdural hematoma: a decision analysis. J Neurosurg. (2010) 113:615-21. doi: 10.3171/2009.9.JNS08825 
11. Hoffman H, Jalal MS, Bunch KM, Chin LS. Management of chronic subdural hematoma with the subdural evacuating port system: systematic review and meta-analysis. J Clin Neurosci. (2021) 86:154-63. doi: 10.1016/j.jocn.2021.01.039

12. Chari A, Kolias AG, Santarius T, Bond S, Hutchinson PJ. Twist-drill craniostomy with hollow screws for evacuation of chronic subdural hematoma. J Neurosurg. (2014) 121:176-83. doi: 10.3171/2014.4.JNS131212

13. Golub D, Ashayeri K, Dogra S, Lewis A, Pacione D. Benefits of the subdural evacuating port system (SEPS) procedure over traditional craniotomy for subdural hematoma evacuation. Neurohospitalist. (2020) 10:257-65. doi: 10.1177/1941874420920520

14. Wang K, Chen D, Cao X, Gao L. A prospective comparative study of twist drill craniostomy versus burr hole craniostomy in patients with chronic subdural hematoma. Turk Neurosurg. (2017) 27:60-5. doi: 10.1186/s12893-017-0295-x

15. Muzii VF, Bistazzoni S, Zalaffi A, Carangelo B, Mariottini A, Palma L. Chronic subdural hematoma: comparison of two surgical techniques. Preliminary results of a prospective randomized study. J Neurosurg Sci. (2005) 49:41-6.

16. Rughani AI, Lin C, Dumont TM, Penar PL, Horgan MA, Tranmer BI. A case-comparison study of the subdural evacuating port system in treating chronic subdural hematomas. J Neurosurg. (2010) 113:60914. doi: 10.3171/2009.11.JNS091244

17. Singh SK, Sinha M, Singh VK, Parihar A, Srivastava C, Ojha BK, et al. A randomized study of twist drill versus burr hole craniostomy for treatment of chronic subdural hematomas in 100 patients. Indian J Neurotrauma. (2011) 8:83-8. doi: 10.1016/S0973-0508(11)80005-5

18. Szmuda T, Kierońska S, Słoniewski P, Dzierzanowski J. Modified bedside twist drill craniostomy for evacuation of chronic subdural haematoma. Wideochir Inne Tech Maloinwazyjne. (2019) 14:442-50. doi: 10.5114/wiitm.2019.83001

19. Wang QF, Cheng C, You C. A new modified twist drill craniostomy using a novel device to evacuate chronic subdural hematoma. Medicine (Baltimore). (2016) 95:e3036. doi: 10.1097/MD.0000000000003036

20. Xu C, Chen B, Xue L, Xia L, Yang X, Wei M, et al. Randomized controlled study on the curative effects of twist-drill craniotomy and burrhole craniotomy in the treatment of chronic subdural hematoma. Exp Ther Med. (2018) 16:959-65. doi: 10.3892/etm.2018.6265

21. Teng JQ. Clinical comparison of the treatment of chronic subdural hematoma by pulverizing intracranial hematoma with puncture needle and drilling hematoma drainage in skull. Shenzhen J Integrated Traditional Chin Western Med. (2017) 27:15-6.(in-Chinese).

22. Liu JG, Ge HT, Yan XH. Therapeutic effect of burr-hole craniostomy with closed-system drainage and drainage with YL-1 puncture needle on chronic subdural hematoma. Chin Neurosurg J. (2004) 3:134.(in-Chinese).

23. Zhu $\mathrm{ZH}$, Wang XD. Treatment of chronic subdural hematoma by trepanation and drainage fluid contrast with YL-1minimally invasive transcutaneous puncture. Chin J Injury Repair Wound Healing. (2010) 5:354-8.(in-Chinese).

24. Flint AC, Chan SL, Rao VA, Efron AD, Kalani MA, Sheridan WF. Treatment of chronic subdural hematomas with subdural evacuating port system placement in the intensive care unit: evolution of practice and comparison with bur hole evacuation in the operating room. J Neurosurg. (2017) 127:14438. doi: 10.3171/2016.9.JNS161166

25. Ortiz M, Belton P, Burton M, Litofsky NS. Subdural drain versus subdural evacuating port system for the treatment of nonacute subdural hematomas: a single-center retrospective cohort study. World Neurosurg. (2020) 139:e35562. doi: 10.1016/j.wneu.2020.03.226

26. Xu M, Wang WH, Zhu SQ, Tan WG, Jin XG, Lu W, et al. Effects of minimally invasive approaches on chronic subdural hematoma by novel YL-1 puncture needle and burr-hole methods. Acta Neurol Belgica. (2020) 120:37-42. doi: 10.1007/s13760-018-0914-Z
27. Safain M, Roguski M, Antoniou A, Schirmer CM, Malek AM, Riesenburger R. A single center's experience with the bedside subdural evacuating port system: a useful alternative to traditional methods for chronic subdural hematoma evacuation. J Neurosurg. (2013) 118:694-700. doi: 10.3171/2012.11.JNS12689

28. Smely C, Madlinger A, Scheremet R. Chronic subdural haematomaa comparison of two different treatment modalities. Acta Neurochir (Wien). (1997) 139:818-25, discussion 825-6. doi: 10.1007/BF0141 1399

29. Gabarros A, Acebes JJ, Rodriguez R, Cabiol J, Obarrio LL, Marnov $A$, et al. Results of surgical treatment in chronic subdural hematoma Comparison between two techniques: twist-drill and continuous closed drainage versus two burr holes and open external drainage. Neurocirugia. (2000) 11:377. doi: 10.1016/S1130-1473(00)70952-3

30. Balser D, Rodgers SD, Johnson B, Shi C, Tabak E, Samadani U. Evolving management of symptomatic chronic subdural hematoma: experience of a single institution and review of the literature. Neurol Res. (2013) 35:23342. doi: 10.1179/1743132813Y.0000000166

31. Wan Y, Fei X, Jiang D, Chen H, Shi L, Wang Z. Clinical observation of treatment of chronic subdural hematoma with novel double needle minimally invasive aspiration technology. J Craniofac Surg. (2017) 28:6469. doi: 10.1097/SCS.0000000000003462

32. Fei X, Wan Y, Wang Z, Chen H, Jiang D. Application of YL-1 needle in chronic subdural hematoma treatment for super-aged patients. J Craniofac Surg. (2018) 29:e90-4. doi: 10.1097/SCS.0000000000004198

33. Fomchenko EI, Gilmore EJ, Matouk CC, Gerrard JL, Sheth KN Management of subdural hematomas: part II. Surgical management of subdural hematomas. Curr Treat Options Neurol. (2018) 20:34. doi: 10.1007/s11940-018-0518-1

34. Gazzeri R, Galarza M, Neroni M, Canova A, Refice GM, Esposito $\mathrm{S}$. Continuous subgaleal suction drainage for the treatment of chronic subdural haematoma. Acta Neurochir (Wien). (2007) 149:487-93; discussion 493. doi: 10.1007/s00701-007-1139-8

35. Mehta V, Harward SC, Sankey EW, Nayar G, Codd PJ. Evidence based diagnosis and management of chronic subdural hematoma: a review of the literature. J Clin Neurosci. (2018) 50:7-15. doi: 10.1016/j.jocn.2018.01.050

36. Grobelny BT, Ducruet AF, Zacharia BE, Hickman ZL, Andersen KN, Sussman E, et al. Preoperative antiepileptic drug administration and the incidence of postoperative seizures following bur hole-treated chronic subdural hematoma. J Neurosurg. (2009) 111:1257-62. doi: 10.3171/2009.6.JNS0928

Conflict of Interest: The authors declare that the research was conducted in the absence of any commercial or financial relationships that could be construed as a potential conflict of interest.

Publisher's Note: All claims expressed in this article are solely those of the authors and do not necessarily represent those of their affiliated organizations, or those of the publisher, the editors and the reviewers. Any product that may be evaluated in this article, or claim that may be made by its manufacturer, is not guaranteed or endorsed by the publisher.

Copyright (c) 2022 Wei, Jiang, Wang and Wang. This is an open-access article distributed under the terms of the Creative Commons Attribution License (CC BY). The use, distribution or reproduction in other forums is permitted, provided the original author(s) and the copyright owner(s) are credited and that the original publication in this journal is cited, in accordance with accepted academic practice. No use, distribution or reproduction is permitted which does not comply with these terms. 